\title{
Transcendental values of the $p$-adic digamma function
}

\author{
by \\ M. Ram Murty (Kingston) and N. SARAdHa (Mumbai) \\ In honour of Professor Wolfgang Schmidt
}

1. Introduction. Let $\psi(x)$ denote the digamma function. This is the logarithmic derivative of Euler's $\Gamma$-function. It is known that $\psi(1)=-\gamma$, where $\gamma$ is Euler's constant. The study of the values of the digamma function at rational arguments was the focus of our earlier paper [8]. There, we showed that at most one of the numbers in the list:

$$
\gamma, \psi(r / q), \quad \operatorname{gcd}(r, q)=1,1 \leq r \leq q,
$$

is algebraic. It is reasonable to conjecture that all of the above numbers are transcendental.

In a related context, Lehmer [6] defined generalized Euler constants $\gamma(r, q)$ by the formula

$$
\gamma(r, q):=\lim _{x \rightarrow \infty}\left(\sum_{n \leq x, n \equiv r(\bmod q)} \frac{1}{n}-\frac{\log x}{q}\right) .
$$

One expects that all of these generalized Euler constants are transcendental. In [8], it was shown that at most one of the numbers

$$
\gamma, \gamma(r, q), \quad \operatorname{gcd}(r, q)=1,1 \leq r \leq q,
$$

is algebraic.

When we obtained this result, Professor Waldschmidt indicated that it would be interesting to investigate the arithmetic nature of the $p$-adic analogues of these numbers. There are two different definitions available in the literature, one due to Morita [7] and another due to Diamond [4]. The $p$-adic analogues of the digamma function and Euler's constant defined by Diamond possess several nice properties as in the classical case. Thus, one

2000 Mathematics Subject Classification: Primary 11J86; Secondary 11J99.

Key words and phrases: $p$-adic digamma function, $p$-adic Euler's constant, transcendence, linear forms in $p$-adic logarithms.

Research of M. R. Murty partially supported by an NSERC grant. 
may expect that arithmetic results similar to the ones stated above also hold in the $p$-adic case. This turns out to be the case and the precise statement is given in Theorem 1. Though there are similarities in the set up, there are new technical difficulties to be overcome. As in the "classical" case, the proof depends on the $p$-adic analogue of Baker's theory of linear forms in logarithms. Unlike the classical case, it involves a clever application of the elementary Euler-Fermat theorem (see Section 3). Further, one needs to find a suitable set of multiplicatively independent units in a cyclotomic field. Our hope is that this study will eventually lead to a proof of the transcendence of the $p$-adic Euler constant as well as shed some light on the classical Euler constant. Indeed, Proposition 5 shows that there is a relationship between the $p$-adic digamma function and the classical digamma function. Thus, it is not unreasonable to study carefully the $p$-adic setting and see what generalizes and what does not.

Next, we turn our attention to $L_{p}(s, f)$, the $p$-adic analogue of the classical $L$-function $L(s, f)$ studied in [8]. The study of these $p$-adic $L$-functions is very much in the spirit of Kubota, Leopoldt and Iwasawa. Before we proceed, it may help the reader if we say a few words about the larger relevance of such a study.

In the second half of the 20th century, Kubota, Leopoldt and Iwasawa introduced the $p$-adic analogue of the classical Dirichlet $L$-function. The study of the $p$-adic $L$-functions forms the basis of what is now called Iwasawa theory. Since then, many authors have introduced $p$-adic analogues of $L$-series attached to modular forms or more generally to automorphic forms. These $L$-series have propelled the study of higher dimensional analogues of Iwasawa's theory. This motif has been useful in the study of special values of $L$-series and their arithmetic meaning. We envisage a similar application of the results we are about to derive. As in the classical case, the non-vanishing of $L_{p}(1, f)$ is an important question. In this direction, Okada [10] showed a connection between the non-vanishing of $L(1, f)$ and $L_{p}(1, f)$ (see Proposition 5). Our idea is to bring forth the utility of this result along with several criteria that we gave in [8] for the non-vanishing of $L(1, f)$. For instance, we are able to show the transcendence of $L_{p}(1, f)$ (see Theorem 6$)$.

In [8], we showed that the vanishing of a certain $L$-function at $s=1$ was equivalent to the existence of relations among special values of the digamma function. In a similar vein, the vanishing of a certain $p$-adic $L$-function will imply relations among special values of the $p$-adic digamma function.

Let $p$ be a prime number which will be fixed throughout this paper once and for all. Let $\mathbb{Q}_{p}$ denote the $p$-adic completion of the rationals $\mathbb{Q}$, and $\mathbb{Z}_{p}$ the $p$-adic completion of the integers $\mathbb{Z}$. Let $\mathbb{C}_{p}$ be the completion of the algebraic closure of $\mathbb{Q}_{p}$. 
In 1975, Morita [7] introduced the $p$-adic analogue $\Gamma_{p}$ of the $\Gamma$-function by defining for all natural numbers

$$
\Gamma_{p}(n):=\prod_{1 \leq t \leq n, p \nmid t} t,
$$

and extending it to a continuous function on $\mathbb{Z}_{p}$. Two years later, Diamond [4] defined the $p$-adic analogues of the digamma function and Euler's constant. We denote these by $\psi_{p}(x)$ and $\gamma_{p}$, respectively. He also defined $p$-adic analogues of the generalized Euler constants, $\gamma_{p}(r, q)$. We will recall these definitions below in Section 2.

We will prove:

TheOREM 1. Let $q$ be prime. Then at most one of the numbers

$$
\gamma_{p}, \gamma_{p}(r, q), \quad 1 \leq r<q,
$$

is algebraic.

As discussed in [4], there are several ways to define a $p$-adic analogue of the digamma function. One way is as follows. Let $\nu(x)$ denote the $p$-adic valuation with $\nu(p)=1$ and $\log$ be the $p$-adic logarithm (defined below in Section 2). Let

$$
H_{N}(x)=\lim _{k \rightarrow \infty} \frac{1}{p^{k}} \sum_{n=0}^{p^{k}-1} f_{N}(x+n)
$$

where $f_{N}(x)=x \log x-x$ if $\nu(x)<N$ and zero otherwise. One can show that $H_{N}$ is locally analytic on $\mathbb{C}_{p}$. For $x \in \mathbb{C}_{p} \backslash \mathbb{Z}_{p}$, the sequence of values $H_{N}(x)$ with $N \geq 1$ eventually becomes constant with the value $G_{p}(x)$ (say), and this is Diamond's $p$-adic analogue of the logarithm of the $\Gamma$-function. Following [4], we denote the $p$-adic derivative of $G_{p}(x)$ by $\psi_{p}(x)$. One can also view the functions $H_{N}$ as suitable $p$-adic analogues of the logarithm of the classical $\Gamma$-function. For instance, $H_{1}$ is the logarithm of Morita's $p$-adic gamma function. With this understanding, we prove:

TheOREM 2. Let q be prime.

(i) If $q=p$, the numbers $\psi_{p}(r / q)+\gamma_{p}$ are transcendental for $1 \leq r<q$.

(ii) If $q \neq p$, let $N$ satisfy the congruence $p^{N} \equiv 1(\bmod q)$. Then the numbers

$$
\frac{p^{N}}{p^{N}-1} H_{N}^{\prime}(r / q)+\gamma_{p}
$$

are transcendental for $1 \leq r<q$.

This theorem can be viewed as the $p$-adic analogue of a result of Bundschuh [3] that states that all the numbers

$$
\psi(a / q)+\gamma, \quad \operatorname{gcd}(a, q)=1,1 \leq a \leq q,
$$


are transcendental. In [8], we rediscovered this result independently and derived it as a consequence of a theorem of Baker, Birch and Wirsing [2].

In this paper, our approach will be analogous to the one we took in [8]. There, we used the theory of the Hurwitz zeta function to deduce a variety of results about the values of the digamma function. Here too, we will use the $p$-adic analogue of the Hurwitz zeta function as defined by Washington [12]. As in [8], let $f$ be a function defined on the non-negative integers, periodic with period $q$ and assuming algebraic values. We denote by $\hat{f}$ the Fourier transform of $f$. Recall that this is given by

$$
\hat{f}(n)=\frac{1}{q} \sum_{a=1}^{q} f(a) e^{2 \pi i a n / q} .
$$

We will say $f$ is of Dirichlet type if $f(a)=0$ whenever $\operatorname{gcd}(a, q)>1$. Inspired by a question of Chowla, the result of Baker, Birch and Wirsing [2] gave necessary conditions for the non-vanishing of the infinite series

$$
L(s, f)=\sum_{n=1}^{\infty} \frac{f(n)}{n^{s}}
$$

at $s=1$. In [9], we gave a variant of their result. We combine these two results as follows.

Proposition 3. Let $K$ be an algebraic number field with $K \cap \mathbb{Q}\left(\zeta_{q}\right)=\mathbb{Q}$ where $\zeta_{q}$ is a primitive qth root of unity. Assume that $f$ is a $K$-valued periodic function with period $q$ and not identically zero. Then $L(1, f) \neq 0$ if $f(n)=0$ for $1<\operatorname{gcd}(n, q)<q$. If $\hat{f}$ is of Dirichlet type and $q$ is a prime power, then $L(1, f) \neq 0$. If $\hat{f}$ is of Dirichlet type and $q$ is not a prime power, then $L(1, f) \neq 0$ unless $f=\lambda c_{q}$ for some $\lambda \in K$ where $c_{q}(n)$ denotes the Ramanujan sum

$$
\sum_{(a, q)=1} e^{2 \pi i a n / q}
$$

In this context, we point out that Okada [10] gave necessary and sufficient conditions under which $L(1, f) \neq 0$. Since these conditions are unwieldy, we prefer to use Proposition 3, as the conditions stated there are more transparent. It will be fruitful to make Okada's conditions more lucid for applications.

Thanks to Baker's theory of linear forms in logarithms, the non-vanishing of $L(1, f)$ allows us to deduce that it is transcendental. Thus, the nonvanishing is the essential ingredient and the above proposition allows us to deduce this in certain cases. For the purpose of this paper, we need only consider even functions $f$. In this case it was shown in the proof of Theorem 3 of [2] that the condition $K \cap \mathbb{Q}\left(\zeta_{q}\right)=\mathbb{Q}$ is redundant. Note 
that this condition is equivalent to the $q$ th cyclotomic polynomial being irreducible over $K$. Thus, we have the following proposition.

Proposition 4. Let $K$ be an algebraic number field and $f$ a $K$-valued periodic even function with period $q$ and not identically zero. Then $L(1, f)$ $\neq 0$ if $f(n)=0$ for $1<\operatorname{gcd}(n, q)<q$. If $\hat{f}$ is of Dirichlet type and $q$ is a prime power, then $L(1, f) \neq 0$. If $\hat{f}$ is of Dirichlet type and $q$ is not a prime power, then $L(1, f) \neq 0$ unless $f=\lambda c_{q}$ for some $\lambda \in K$ where $c_{q}(n)$ denotes the Ramanujan sum.

We now discuss $p$-adic analogues of the above proposition. Proceeding as in [8], one defines the $p$-adic $L$-function $L_{p}(s, f)$ as a linear combination of $p$ adic Hurwitz zeta functions. From this definition, it follows that $L_{p}(s, f) \equiv 0$ if $f$ is an odd function. So henceforth, we assume that $f$ is an even function. Now we present Theorem 2 of Okada [10] in which he connects the nonvanishing of $L(1, f)$ and $L_{p}(1, f)$.

Proposition 5. Let $f$ be an even function which is algebraic-valued and with period $q$. Put $\tilde{f}=f \chi_{0}$ where $\chi_{0}$ is the principal character mod $p$. Then $L_{p}(1, f)=0$ if and only if $L(1, \tilde{f})=0$. Further, if $p \nmid q$, then $L_{p}(1, f)=0$ if and only if $L(1, f)=0$.

Proceeding as in [8], $L_{p}(1, f)$ can be expressed as a linear form in $p$-adic logarithms of algebraic numbers with algebraic coefficients. Baker's theory has been extended to the $p$-adic context by several people. In this setting, we employ a result of Kaufman [5] (see also [11]), together with Proposition 5 to obtain the following two results.

THEOREM 6. Let $q$ be a prime and $f$ an algebraic-valued even function with period $q$. Then $L_{p}(1, f)$ is transcendental.

TheOREM 7. Let $q$ be greater than 1 and $K$ an algebraic number field. Suppose that $f$ is a $K$-valued even function with period $q$ and that $L_{p}(s, f)$ is regular at $s=1$. Suppose further that $p \mid q$ and one of the following conditions holds:

(i) $\tilde{f}(n)=0$ whenever $1<\operatorname{gcd}(n, q)<q$;

(ii) the Fourier transform of $\tilde{f}$ is of Dirichlet type and $\tilde{f} \neq \lambda c_{q}$ for any $\lambda \in K$.

Then $L_{p}(1, f)$ is transcendental.

Suppose that $p \nmid q$ and one of the following conditions holds:

(iii) $f(n)=0$ for $1<\operatorname{gcd}(n, q)<q$;

(iv) $\hat{f}$ is of Dirichlet type and $f \neq \lambda c_{q}$ for any $\lambda \in K$.

Then $L_{p}(1, f)$ is transcendental.

As a consequence of Theorem 7 , we get: 
COROLlary 8. The values of $L_{p}(1, \chi)$ as $\chi$ ranges over even Dirichlet characters mod $q$ are linearly independent over $\overline{\mathbb{Q}}$. Any non-trivial linear combination of these values is transcendental.

Combining this corollary with Proposition 5 gives:

COROLlary 9. Let $q$ be prime and $\chi$ range over even non-trivial Dirichlet characters mod $q$. Then the $L(1, \chi)$ are linearly independent over $\overline{\mathbb{Q}}$.

Corollary 9 was proved earlier by Baker, Birch and Wirsing [2] using Baker's theory of linear forms in logarithms. By combining both the $p$-adic theory and the classical theory of linear forms in logarithms, Okada [10] shows that if $\chi$ runs through all the non-principal even Dirichlet characters $\bmod N$ and $\ell$ runs through all the prime factors of $N$, then the numbers $L(1, \chi)$ and $\log \ell$ are linearly independent over $\overline{\mathbb{Q}}$.

In the next section, we review the definitions of the $p$-adic analogues of the digamma function, Euler's constant and the $L$-function to set the stage for the proofs of the above theorems.

2. Preliminaries. $\mathbb{C}_{p}$ is the $p$-adic analogue of the complex numbers. We fix once and for all an embedding of $\overline{\mathbb{Q}}$ into $\mathbb{C}_{p}$. An element of $\mathbb{C}_{p} \backslash \overline{\mathbb{Q}}$ is called transcendental. We denote by $B_{n}$ the $n$th Bernoulli number given by the coefficient of $t^{n}$ in the power series expansion of

$$
\frac{t e^{t}}{e^{t}-1} \text {. }
$$

Let $v$ denote the $p$-adic valuation in $\mathbb{C}_{p}$ with $v(p)=1$ and denote by $|\cdot|$ the $p$-adic metric with the normalization $|p|=1 / p$. Lehmer [6] defined generalized Euler constants as follows. Let $r, q$ be positive integers with $r \leq q$. Then

$$
\gamma(r, q)=\lim _{x \rightarrow \infty}\left(\sum_{n \leq x, n \equiv r(\bmod q)} \frac{1}{n}-\frac{1}{q} \log x\right) .
$$

One can show that

$$
\psi(r / q)=\log q-q \gamma(r, q) .
$$

When $r=q=1, \gamma(1,1)=\gamma$ is the classical Euler constant and $\psi(1)=-\gamma$. In $\mathbb{C}_{p}$, the analogous $\gamma_{p}(r, q)$ is defined as follows (see Diamond [4] for details and motivation). We define the $p$-adic logarithm as

$$
\log _{p}(1+x)=x-x^{2} / 2+x^{3} / 3-x^{4} / 4+\cdots
$$

which is valid for $|x|_{p}<1$. We refer the reader to [12] for further details concerning the $p$-adic logarithm and its extension to $\mathbb{C}_{p}^{\times}$. We will henceforth write $\log$ for the $p$-adic logarithm. Let $r, q$ be integers with $q \geq 1$. Suppose 
that $v(r / q)<0$. Define

$$
\gamma_{p}(r, q)=-\lim _{k \rightarrow \infty} \frac{1}{q p^{k}} \sum_{m \geq 1, m \equiv r(\bmod q)}^{q p^{k}-1} \log m .
$$

If $v(r / q) \geq 0$, we write $q=p^{k} q^{*}$ with $\left(p, q^{*}\right)=1$ and define

$$
\gamma_{p}(r, q)=\frac{p^{\phi\left(q^{*}\right)}}{p^{\phi\left(q^{*}\right)-1}} \sum_{n \in N(r, q)} \gamma_{p}\left(r+n q, p^{\phi\left(q^{*}\right)} q\right),
$$

where

$$
N(r, q)=\left\{n: 0 \leq n<p^{\phi\left(q^{*}\right)}, r+n q \not \equiv 0\left(\bmod p^{\phi\left(q^{*}\right)+k}\right)\right\} .
$$

We set

$$
\gamma_{p}=\gamma_{p}(0,1)=-\frac{p}{p-1} \lim _{k \rightarrow \infty} \frac{1}{p^{k}} \sum_{m \geq 1,(m, p)=1}^{p^{k}-1} \log m .
$$

Define

$$
\psi_{p}(x)=\lim _{k \rightarrow \infty} \frac{1}{p^{k}} \sum_{n=0}^{p^{k}-1} \log (x+n) \quad \text { for any } x \in \mathbb{C}_{p} .
$$

In Theorem 18 of [4], the following result is established.

Lemma 10. If $q>1$ and $\zeta$ is a primitive $q$ th root of unity, then

$$
q \gamma_{p}(r, q)=\gamma_{p}-\sum_{a=1}^{q-1} \zeta^{-a r} \log \left(1-\zeta^{a}\right)
$$

From the definition of $\gamma_{p}(r, q)$ in (2), the first part of the following lemma is immediate. The second part is proved in [4] and appears there as Theorem 19.

Lemma 11. Suppose $r, q$ are positive integers with $r<q$ and $v(r / q)<0$. Then

$$
\psi_{p}(r / q)=-\log q-\gamma_{p}+\sum_{a=1}^{q-1} \zeta^{-a r} \log \left(1-\zeta^{a}\right),
$$

where $\zeta$ denotes a primitive $q$ th root of unity. Write $q=p^{k} q^{*}$ with $p$ and $q^{*}$ coprime. If $\nu(r / q) \geq 0$, then for any $N$ such that $p^{N} \equiv 1\left(\bmod q^{*}\right)$, we have

$$
\frac{p^{N}}{p^{N}-1} H_{N}^{\prime}(r / q)=-\log q-\gamma_{p}+\sum_{a=1}^{q-1} \zeta^{-a r} \log \left(1-\zeta^{a}\right) .
$$

We now proceed to define the $p$-adic $L$-function. We refer to Washington [12] for more details and properties. Let $p^{*}=p$ if $p>2$ and $p^{*}=4$ if $p=2$. 
Suppose $\omega(x)$ is the Dirichlet character with conductor $p^{*}$ such that

$$
\omega(x) \equiv x\left(\bmod p^{*}\right) .
$$

For any $p$-adic unit $x$, let

$$
x=\omega(x)\langle x\rangle .
$$

Let $\mathcal{K}=\operatorname{lcm}\left(p^{*}, q\right)$ and $b$ any integer with $p \nmid b$. The $p$-adic partial zeta function $L_{p}(s, b, \mathcal{K})$ is defined by

$$
L_{p}(s, b, \mathcal{K})=\frac{1}{s-1} \frac{1}{\mathcal{K}}\langle b\rangle^{1-s} \sum_{j=0}^{\infty}\left(\begin{array}{c}
1-s \\
j
\end{array}\right) B_{j}(\mathcal{K} / b)^{j},
$$

where the $B_{j}$ 's denote the Bernoulli numbers. Define

$$
L_{p}(s, f)=\sum_{b=1, p \nmid b}^{\mathcal{K}} f(b) L_{p}(s, b, \mathcal{K}) .
$$

It is shown in [12] that $L_{p}(s, f)$ is analytic on

$$
\left\{s \in \mathbb{C}_{p}:|s|<p^{*} p^{-1 /(p-1)}\right\},
$$

except for a possible simple pole at $s=1$ with residue

$$
R_{p}(f)=\frac{1}{\mathcal{K}} \sum_{b=1, p \nmid b}^{\mathcal{K}} f(b) .
$$

Hence, for $L_{p}(s, f)$ to be regular at $s=1$, it is necessary and sufficient that $R_{p}(f)=0$. Suppose $\chi$ is a non-principal even Dirichlet character $\bmod q$ and $\chi^{*}$ the associated primitive character and $N(\chi)$ the conductor of $\chi^{*}$. Then

$$
L_{p}(1, \chi)=L_{p}\left(1, \chi^{*}\right) \prod_{t \mid q, t \neq p}\left(1-\frac{\chi^{*}(t)}{t}\right) .
$$

By Corollary 5.30 and Theorem 5.18 of [12], it follows that $L_{p}(1, \chi) \neq 0$ and

$$
L_{p}(1, \chi)=c(\chi) \sum_{r=1}^{N(\chi)} \bar{\chi}^{*}(r) \log \left(1-\zeta^{r q / N(\chi)}\right),
$$

where $c(\chi)$ is an algebraic number and $\zeta$ is a primitive $q$ th root of unity. Okada (see (3.8) of [10]) has shown that if $f(b) \in K$, then

$$
L_{p}(1, f)=\sum_{\chi} \delta_{\chi} L_{p}(1, \chi)+\sum_{t \mid q, t \neq p} \mu_{t} \log t,
$$

where $\delta_{\chi}$ and $\mu_{t}$ are in $K$ and $\chi$ runs over all non-principal even characters $\bmod q$. Together with $(3)$ and $(4)$, we see that $L_{p}(1, f)$ can be expressed as a linear form in $p$-adic logarithms of algebraic numbers with algebraic coefficients. There have been several papers on quantitative results in the theory of linear forms in $p$-adic logarithms, the most notable being by $\mathrm{Yu}$ 
[13]. Since for the purpose of this paper we need only qualitative results, we use one of the earliest results by Kaufman [5] in this direction.

Lemma 12. Let $\alpha_{1}, \ldots, \alpha_{m}$ be fixed algebraic numbers that are multiplicatively independent over $\mathbb{Q}$ with height at most $h$. Let $\beta_{0}, \ldots, \beta_{m}$ be arbitrary algebraic numbers with height at most $H$ (assumed greater than 1) and $\beta_{0} \neq 0$. There exists a constant $c_{1}>0$ which depends only on the degree of the number field generated by $\alpha_{1}, \ldots, \alpha_{m}, \beta_{0}, \beta_{1}, \ldots, \beta_{m}$ such that the following holds. Let $K=\mathbb{Q}\left(\alpha_{1}, \ldots, \alpha_{m}, \beta_{0}, \beta_{1}, \ldots, \beta_{m}\right)$ and $\left|\alpha_{i}-1\right|<p^{-c_{1}}$ for $1 \leq i \leq m$. Then

$$
\left|\beta_{0}+\beta_{1} \log \alpha_{1}+\cdots+\beta_{m} \log \alpha_{m}\right|>p^{-c \log H}
$$

where $c$ is a constant depending only on $p, h, m$ and $[K: \mathbb{Q}]$.

In particular, if $\alpha_{1}, \ldots, \alpha_{m}$ are algebraic numbers which are multiplicatively independent over $\mathbb{Q}$, then there exists a $c_{1}>0$ as in Lemma 12 such that $1, \log \alpha_{1}, \ldots, \log \alpha_{m}$ are linearly independent over $\overline{\mathbb{Q}}$ whenever $\left|\alpha_{i}-1\right|<p^{-c_{1}}$ for $1 \leq i \leq m$.

As a consequence of Lemma 12, we get

COROLlary 13. Suppose $\alpha_{1}, \ldots, \alpha_{m}$ are non-zero algebraic numbers satisfying $\left|\alpha_{i}-1\right|<p^{-c_{1}}$ for $1 \leq i \leq m$, with $c_{1}$ given by Lemma 12. Then, for any algebraic numbers $\beta_{1}, \ldots, \beta_{m}$, the linear form

$$
\beta_{1} \log \alpha_{1}+\cdots+\beta_{m} \log \alpha_{m}
$$

is either zero or transcendental. The former can arise only if $\alpha_{1}, \ldots, \alpha_{m}$ are multiplicatively dependent.

The proof (by induction on $m$ ) of this theorem is identical to the complex case discussed on page 11 of [1].

In the proof of Theorem 1 below, we will need to apply the following result from the theory of cyclotomic fields.

Lemma 14. Let $q$ be a prime and let $\zeta$ be a primitive qth root of unity. The numbers $1-\zeta$ and

$$
\frac{1-\zeta^{a}}{1-\zeta}, \quad 1<a<q / 2,
$$

are multiplicatively independent.

Proof. The numbers

$$
\frac{1-\zeta^{a}}{1-\zeta}, \quad 1<a<q / 2,
$$

comprise a multiplicatively independent system of units in $\mathbb{Q}(\zeta)$. Thus, if

$$
(1-\zeta)^{b} \prod_{1<a<q / 2}\left(\frac{1-\zeta^{a}}{1-\zeta}\right)^{n_{a}}=1
$$


for certain integers $n_{a}, b$, then, as $(q)=(1-\zeta)^{q-1}$, and $(1-\zeta)$ is a prime ideal, we must have $b=0$ since the right hand side has no prime divisor. Consequently, $n_{a}=0$ for all $a$ because the units in the product are independent.

3. Proof of Theorem 1. Let $q$ be prime. Suppose first that $\gamma_{p}$ and $\gamma_{p}(r, q)$ are both algebraic. Then $q \gamma_{p}(r, q)-\gamma_{p}$ is algebraic. By Lemma 10, this is equal to

$$
-\sum_{a=1}^{q-1} \zeta^{-a r} \log \left(1-\zeta^{a}\right)
$$

Since

$$
\sum_{a=1}^{q-1} \zeta^{-a r}=-1
$$

we rewrite this sum as

$$
\log (1-\zeta)-\sum_{a=1}^{q-1} \zeta^{-a r} \log \left(\frac{1-\zeta^{a}}{1-\zeta}\right)
$$

Since

$$
1-\zeta^{-a}=-\zeta^{-a}\left(1-\zeta^{a}\right)
$$

and the $p$-adic logarithm is zero on roots of unity, we find that

$$
\log \left(1-\zeta^{-a}\right)=\log \left(1-\zeta^{a}\right) .
$$

Noting that the summands for $a=1$ and $a=q-1$ in (5) are zero, and pairing up $a$ with $-a$, we obtain

$$
q \gamma_{p}(r, q)-\gamma_{p}=\log (1-\zeta)+\sum_{1<a<q / 2}\left(\zeta^{a r}+\zeta^{-a r}\right) \log \left(\frac{1-\zeta^{a}}{1-\zeta}\right)
$$

Observe that for any $y$ with $p \nmid y$,

$$
\log y=\frac{1}{p-1} \log \left(1+\left(y^{p-1}-1\right)\right) .
$$

More generally, we have

$$
\log y=\frac{1}{\phi\left(p^{M}\right)} \log \left(1+\left(y^{\phi\left(p^{M}\right)}-1\right)\right) .
$$

We write the right hand side of (6) as

$$
\begin{aligned}
& \frac{1}{\phi\left(p^{M}\right)} \log \left[1-(1-\zeta)^{\phi\left(p^{M}\right)}-1\right] \\
& \quad+\sum_{1<a<q / 2} \frac{\zeta^{a r}+\zeta^{-a r}}{\phi\left(p^{M}\right)} \log \left[1+\left(\frac{1-\zeta^{a}}{1-\zeta}\right)^{\phi\left(p^{M}\right)}-1\right] .
\end{aligned}
$$


For $y$ coprime to $p$, we have by Euler's theorem,

$$
\left|y^{\phi\left(p^{M}\right)}-1\right|<p^{-M} .
$$

Thus, choosing $M$ sufficiently large, we can use Lemma 12 to deduce that the numbers

$$
1-\zeta, \quad \frac{1-\zeta^{a}}{1-\zeta}, \quad 1<a<q / 2,
$$

are multiplicatively dependent. But this contradicts Lemma 14 . Now suppose that $\gamma_{p}\left(r_{1}, q\right)$ and $\gamma_{p}\left(r_{2}, q\right)$ are both algebraic. We write

$$
q \gamma_{p}\left(r_{1}, q\right)-q \gamma_{p}\left(r_{2}, q\right)=-\sum_{a=1}^{q}\left(\zeta^{-a r_{1}}-\zeta^{-a r_{2}}\right) \log \left(1-\zeta^{a}\right) .
$$

We proceed to rewrite the right hand side as an algebraic linear combination of

$$
\log (1-\zeta), \quad \log \left(\frac{1-\zeta^{a}}{1-\zeta}\right), \quad 1<a<q / 2,
$$

as before and deduce the desired result analogously.

It should be evident that the above proof implies the following.

COROllary 15. Let $q>1$ and $r$ be positive integers with $r<q$. Then the numbers

$$
q \gamma_{p}(r, q)-\gamma_{p}
$$

are transcendental.

4. Proof of Theorem 2. As in the proof of Theorem 1, our proof of Theorem 2 will make use of the fact that

$$
\frac{1-\zeta^{a}}{1-\zeta}, \quad 1<a<q / 2,
$$

is a system of fundamental units for the field $\mathbb{Q}(\zeta)$.

First suppose that $q=p$. By the first part of Lemma 11, whenever $p \nmid r$, we have

$$
\psi_{p}(r / q)=-\log q-\gamma_{p}+\sum_{a=1}^{q-1} \zeta^{-a r} \log \left(1-\zeta^{a}\right),
$$

where $\zeta$ is a primitive $q$ th root of unity. Now,

$$
q=\prod_{a=1}^{q-1}\left(1-\zeta^{a}\right)
$$


Therefore,

$$
\log q=\sum_{a=1}^{q-1} \log \left(\frac{1-\zeta^{a}}{1-\zeta}\right)+(q-1) \log (1-\zeta)
$$

Hence,

$$
\psi_{p}(r / q)+\gamma_{p}=-q \log (1-\zeta)-\sum_{a=1}^{q-1}\left(1-\zeta^{-a r}\right) \log \left(\frac{1-\zeta^{a}}{1-\zeta}\right) .
$$

As in the proof of Theorem 1, we can rewrite the sum by pairing up $a$ with $-a$ and get

$$
\psi_{p}(r / q)+\gamma_{p}=-q \log (1-\zeta)-\sum_{1<a<q / 2}\left(2-\zeta^{-a r}-\zeta^{a r}\right) \log \left(\frac{1-\zeta^{a}}{1-\zeta}\right) .
$$

By Lemma 14, the right hand side is non-zero. Again, as in the proof of Theorem 1, we rewrite the right hand side to get

$$
\begin{aligned}
\psi_{p}(r / q)+\gamma_{p}= & -\frac{q}{\phi\left(p^{M}\right)} \log \left[1+\left((1-\zeta)^{\phi\left(p^{M}\right)}-1\right)\right] \\
& -\sum_{1<a<q / 2} \frac{2-\zeta^{a r}-\zeta^{-a r}}{\phi\left(p^{M}\right)} \log \left[1+\left(\frac{1-\zeta^{a}}{1-\zeta}\right)^{\phi\left(p^{M}\right)}-1\right] .
\end{aligned}
$$

Proceeding as before, we deduce from Lemma 14 and Corollary 13 that $\psi_{p}(r / q)+\gamma_{p}$ is transcendental. This completes the proof of the first part of the theorem. The second part is proved in a completely analogous fashion and so we suppress the details.

5. Proof of Theorem 6. Let $q^{*}=q$ if $p=q$ and $q^{*}=p q$ if $p \neq q$. By Proposition $5, L_{p}(1, f)=0$ if and only if $L(1, \tilde{f})=0$. Now, $\tilde{f}$ is a periodic, algebraic-valued function with period $q^{*}$. By its very definition, $\tilde{f}(n)=0$ whenever $1<\operatorname{gcd}(n, q)<q$. Hence by Proposition $3, L(1, \tilde{f}) \neq 0$. Thus, $L_{p}(1, f) \neq 0$. On the other hand, by Washington [12], we have

$$
L_{p}(1, f)=\sum_{r=1}^{q-1} a_{r} \log \left(1-\zeta^{r}\right)+a_{0} \log q,
$$

with $a_{i} \in \mathbb{Q}_{p}(\zeta)$. Now,

$$
\log q=\sum_{r=1}^{q-1} \log \left(1-\zeta^{r}\right)
$$

so that the previous expression can be rewritten simply as an algebraic linear combination of $\log \left[\left(1-\zeta^{r}\right) /(1-\zeta)\right]$ with $1<r \leq q / 2$ and $\log (1-\zeta)$. Since $q$ is prime, these numbers are multiplicatively independent by Lemma 14 . Hence, by Corollary 13, we deduce the transcendence of $L_{p}(1, f)$. 
6. Proof of Theorem $\mathbf{7}$ and Corollary 8. By Proposition 5, we know that $L_{p}(1, f)=0$ if and only if $L(1, \tilde{f})=0$ and further, if $p \nmid q$, that $L_{p}(1, f)=0$ if and only if $L(1, f)=0$. The conditions of Proposition 4 imply that $L(1, \tilde{f}) \neq 0$. Thus, $L_{p}(1, f) \neq 0$.

As seen earlier, $L_{p}(1, f)$ is an algebraic linear combination of $\log \left(1-\zeta^{r}\right)$, $1 \leq r \leq q-1$. Hence, by Corollary 13, we deduce the transcendence of $L_{p}(1, f)$ and this completes the proof.

7. Concluding remarks. Since the function $H_{1}$ is the logarithm of Morita's $p$-adic $\Gamma$-function, one can specialize our results above to write that if $q$ is prime and $p \equiv 1(\bmod q)$, then for $1 \leq r<q$,

$$
\left(1-\frac{1}{p}\right)^{-1} \frac{\Gamma_{p}^{\prime}}{\Gamma_{p}}\left(\frac{r}{q}\right)+\gamma_{p}
$$

is transcendental. This statement has the aesthetic virtue of resembling our earlier result on the classical digamma function. However, in the $p$-adic domain, as is often the case, there are various ways of extending the classical situation.

Acknowledgements. We would like to thank Sanoli Gun and Purusottam Rath for their comments on an earlier version of this paper.

\section{References}

[1] A. Baker, Transcendental Number Theory, 2nd ed., Cambridge Univ. Press, 1990.

[2] A. Baker, B. Birch and E. Wirsing, On a problem of Chowla, J. Number Theory 5 (1973), 224-236.

[3] P. Bundschuh, Zwei Bemerkungen über transzendente Zahlen, Monatsh. Math. 88 (1979), 293-304.

[4] J. Diamond, The p-adic log gamma function and p-adic Euler constants, Trans. Amer. Math. Soc. 233 (1977), 321-337.

[5] R. M. Kaufman, An estimate of a linear form of logarithms of algebraic numbers in a p-adic metric, Vestnik Moskov. Univ. Ser. I Mat. Mekh. 261 (1971), no. 2, 3-10 (in Russian).

[6] D. H. Lehmer, Euler constants for arithmetical progressions, Acta Arith. 27 (1975), 125-142. (See also Selected Papers of D. H. Lehmer, Vol. 2, 591-608.)

[7] Y. Morita, A p-adic analogue of the $\Gamma$-function, J. Fac. Sci. Univ. Tokyo Sect. IA Math. 22 (1975), 255-266.

[8] M. Ram Murty and N. Saradha, Transcendental values of the digamma function, J. Number Theory 125 (2007), 298-318.

[9] - - - Special values of the polygamma functions, Int. J. Number Theory, to appear.

[10] T. Okada, Dirichlet series with periodic algebraic coefficients, J. London Math. Soc. 33 (1986), 13-21.

[11] A. J. van der Poorten, Linear forms in logarithms in the p-adic case, in: Transcendence Theory: Advances and Applications, A. Baker and D. W. Masser (eds.), Academic Press, 1977, 29-57. 
[12] L. Washington, Introduction to Cyclotomic Fields, Springer, 1975.

[13] K. Yu, Report on p-adic logarithmic forms, in: A Panorama of Number Theory or the View from Baker's Garden (Zürich, 1999), Cambridge Univ. Press, Cambridge, 2002, 11-25.

Department of Mathematics

Queen's University

Kingston, Ontario, K7L 3N6, Canada

E-mail: murty@mast.queensu.ca
School of Mathematics

Tata Institute for Fundamental Research Homi Bhabha Road Mumbai 400 005, India E-mail: saradha@math.tifr.res.in

Received on 29.1.2008

and in revised form on 8.8.2008 